\title{
Über das Schicksal und die Funktion der transplantierten Nebenniere.
}

Von

\author{
Dr. H. Shiota.
}

(Ausgeführt unter der Leitung des Herrn Prof. Dr. A. Kreidl im physiologischen Institut der Wiener Universität.)

Die Transplantation von organischem Gewebe und Organen bzw. Organteilen hat grosses praktisehes sowie wissenschaftliches Interesse. Das praktische Interesse kommt wesentlich in der therapeutischen Anwendung der Transplantation zum Ausdruck, indem verloren gegangenes Gewebe durch anderes zu ersetzen gesucht wird (Haut, Muskel, Knochen, Blutgefässe usw.). 'Eine wissenschaftliche Bedeutung gewann die Transplantation hauptsächlich durch die Lehre von der inneren Sekretion.

Mit der wissenschaftlichen Bearbeitung dieser Frage hängt es zusammen, dass man die Organe mit innerer Sekretion transplantierte, Ist doch geradezu der Umstand, dass ein Organ seine Funktion weiter ausübt, wenn es aus dem normalen Situs herausgebracht und an anderer Stelle eingepflanzt wird, ein Kriterium für die innere sekretorische Tätigkeit desselben.

Es ist daher begreiflich, dass fast alle Organe mit innerer Sekretion transplantiert wurden.

Die Funktion einer transplantierten Drüse mit innerer Sekretion wurde bisher in verschiedener Weise zu erbringen gesucht: entweder dadurch, dass der Symptomenkomplex, der nach Entfernung der Drüse mit innerer Sekretion in Erseheinung tritt, durch Transplantation derselben wieder zum Versehwinden gebracht, oder dass nach vollzogener Transplantation durch Entfernung der transplantierten Organe derselbe wieder hervorgerufen wurde.

In beiden Fällen hat man also die Funktion des transplantierten Organs in indirekter Weise zu erbringen gesucht. 
Ein direkter Weg, sich über das Schicksal und insbesondere über die Funktion des transplantierten Organs zu informieren, wurde eigentlich bisher nicht eingeschlagen. Manchmal wurde wohl eine histologische Untersuchung der verpflanzten und längere Zeit dislozierten Drüsen ausgeführt, doch konnte man durch diese Art von Untersuchung nur erfahren, ob das Organ zugrunde gegangen war oder sein normales histologisches Bild beibehalten hatte. In letzterem Falle also liess sich nur erschliessen, ob es funktionsfähig war.

Professor K reid l gab mir die Anregung, die Funktion von transplantierten Drüsen direkt zu untersuchen, um so eine Möglichkeit zu haben, das Funktionieren des transplantierten Organs während der Dauer der Transplantation zu konstatieren.

Bis jetzt kennt man bloss ein drüsiges Organ, dessen Sekretionstätigkeit sich leicht nachweissen lässt. Man weiss, dass die Nebenniere in dem Adrenalin während ihrer Tätigkeit einen Stoff produziert, welcher vermöge seiner charakteristischen Wirkungen sich leicht erkennen lässt.

Man ist durch den Nachweis vom Vorhandensein oder Feblen des Adrenalins in der Nebenniere jederzeit imstande zu sagen, ob die Nebenniere funktioniert oder nicht. Ich habe mich demnach bei der Prüfung der Funktion von transplantierten Drüseu zunächst auf die Nebenniere allein beschränkt und berichte in folgendem über die Versuche, die darin bestanden, dass $z u$ verschiedenen Zeiten das Vorhandensein des Hauptproduktes der Nebenniere, das Adrenalin, in der transplantierten Nebenniere direkt geprüft wurde.

Gleichzeitig wurde die Nebenniere histologisch untersucht und durch die mikrochemische Reaktion (Chromierung) das Vorhandensein der physiologischen Funktion kontrolliert.

Bevor ich auf meine eigenen Versuche eingehe, möge hier die betreffende Literatur in Kürze wiedergegeben werden.

Canal is ${ }^{1}$ ) hat als erster ein abgetragenes Nebennierenstückchen in die Niere eingepflanzt und gesehen, dass es nekrotisch und resorbiert wurde.

Abelous ${ }^{2}$ ) berichtet, dass er eine Nebenniere vom Frosch zwischen die Fasern des M. ileococcygeus eines anderen Frosches

1) Canalis, Contribution à l'étude du développement et de la pathologie des capsules surrénales. Internat. Monatsschr. f. Anat. u. Physiol. Bd. 4. 1887.

2) Abelous, Essay de greffe des capsules surrénales sur la grenouille. Compt. rend. de la société de Biologie, série IX, t. 4 p. 864.1892. 
Über das Schicksal und die Funktion der transplantierten Nebenniere. 433

transplantiert, und dass der Frosch die nach einem Monat ausgeführte beiderseitige Nebennierenexstirpation überstanden habe. De Dominicis ${ }^{1}$ ) verlagerte bei Hunden die linke Nebenniere mit dem Gefässstiel, der unterbunden wurde, unter die Niere. Obgleich er an der so verlagerten Nebenniere nach 10-12 Tagen keine Veränderungen nachweisen konnte, starb das Tier dennoch nach der Exstirpation des rechten Organs. Gourfe in ${ }^{2}$ ) verpflanzte die Nebenniere vom Froseh und Meerschweinchen in den Lymphsack des Frosches, $\mathrm{B}_{0}$ in $\mathrm{t}^{3}$ ) die der Ratten in die Peritonealhöhle, Hutgren und Andersson ${ }^{4}$ ) die der Katzen und Kaninchen in die Muskeln; bei allen. diesen Versuchen gingen die transplantierten Drüsen zugrunde.

In neuerer Zeit baben verschiedene Autoren die Transplantation von Nebenniere in die blutreichen Organe wie Niere, Leber und Milz ausgeführt [ $\operatorname{Imber\mathrm {t}^{5}}{ }^{5}$, Strehl-Weiss ${ }^{6}$ ), Kreidl ${ }^{7}$ ), Biedl ${ }^{8}$ )]. Sie haben auch gezeigt, dass die transplantierten Organe zugrunde gehen.

Dagegen glauben $\mathrm{P}_{0} \mathrm{ll}^{9}$ ) (bei Ratten), A. und B. Christiani ${ }^{10}$ ) (bei Ratten) und Schmieden ${ }^{11}$ ) (bei Kaninchen), dass ihre Transplantationsversuche mit Nebennieren von Erfolg begleitet waren; sie fanden, dass der Rindenteil des verpflanzten Organs ziemlich lange

1) De Dominicis, Experimentelle Untersuchung zur Physiologie der Nebenniere, Wirkung der Transplantation derselben. Wiener med. Wochenschr. 1897 Nr. 1.

2) Gourfein, Recherches physiologiques sur la fonction des glandes surrénales. Revue med. de la Suisse Romande $1893 \mathrm{Nr} .3$.

3) Boinet, Résultats éloignés de soixante quinze ablations de deux capsules surrénales. Compt. rend. de la société de Biolog. 1895.

4) Hutgren und Andersson, Studien zur Physiologie und Anatomie der Nebenniere. Veittes, Leipzig 1899.

5) Imbert, Recherches expérimentales sur les greffes des capsules surrénales dans la rein. IV. Sess. de la soc. franc. d'urolog. 1899.

6) Strehl und Weiss, Beiträge zur Physiologie der Nebenniere. Pflüger's Arch. Bd. 86 S. 107.

7) Kreid1, Wiener klin. Wochenschr. 1907 S. 615 und 1908 S. 27.

8) Bied1, Wiener klin. Wochenschr. 1907 S. 615.

9) Poll, Veränderungen der Nebenniere bei der Transplantation. Arch. f. mikroskop. Anat. u. Eutwicklungsgeschichte Bd. 54 S. 440. 1899.

10) A. et H. Christiani, De la greffe des capsules surrénales. Journ. de physiol. t. 4.

11) Schmieden, Erfolgreiche experimentelle Verlagerung von Nebennierengewebe. Ein Beitrag zur Lehre von den Strumae suprarenales aberratae. Deutsche Zeitschr. f. Chiurgie Bd. 70. 1908. 
am Leben erhalten werden kann oder sich sogar regeneriert, schliesslich aber doch zugrunde geht.

v. $\mathrm{Haberer}{ }^{1}$ ) hat vor kurzem durch zahlreiche Tierversuche gezeigt, dass die Transplantation der Nebenniere mit Gefässstiel in die Niere in $50 \%$ sowohl anatomisch als auch funktionell einwandfreie Dauererfolge gibt.

Jüngst hat $\mathrm{C}_{0}$ e $\mathrm{n}$ e $\mathrm{n}^{2}$ ) bei einem Hunde 8 Wochen nach der Transplantation der rechten Nebenniere in die Milz die linke exstirpiert. Der Hund starb. Bei fünf Hunden hat er 15 Tage bis 6 Wochen nach der Transplantation der rechten Nebenniere in die Milz oder Niere die linke wieder verpflanzt. Je zwei starben nach 24 und 36 Stunden und einer nach $2^{1 / 2}$ Tagen. Histologisch zeigte die Nebenniere schon nach 24 Stunden Zeichen einer Nekrobiose, und am 5. Tage war schon jede Struktur verloren. Nach 8 Wochen sieht man an Stelle der verpflanzten Nebenniere bloss Pigment in granulierenden Zellen.

Die von mir ausgeführte Methode ist kurz folgende:

Unter aseptischen Kautelen wird einem Versuchstiere (meist Kaninchen und einige Katzen) eine Nebenniere exstirpiert und diese - $\mathrm{n}$ toto oder ein Stück derselben ganz oder geteilt in die Milz verpflanzt; bei einigen Tieren wurde Nebennierengewebe sowohl in die Milz als auch in die Niere eingebracht. Einigemal habe ich auch die Nebenniere eines anderen Tieres derselben Spezies verwendet.

Bei einem Versuchstiere wurde das eine dickere Ende der Milz von einer Kante aus mit einem schmalen Messer eingestochen und ein Schlitz gemacht von der Grösse des kleineren Durchmessers der Nebenniere, dann das Parenchym mit einem kleinen Löffel etwas ausgekratzt, um für die Nebenniere genug Raum zu schaffen, und diese in die Milz hineingeschoben. Durch leichten Druck auf die Milz steht die Blutung; die Nebenniere hält sehr gut, so dass gewöhnlich keine Milznaht notwendig ist; nur in einigen wenigen Fällen wurde eine solche vorgenommen.

Als Einpflanzungsstelle in die Niere wurde der obere Pol derselben gewählt. Hier wurde eine Inzision gemacht, in dieselbe die keilförmig ausgeschnittene Nebenniere eingekeilt, über dieser durch

1) v. Haberer, Experimentelle Verlagerung der Nebenniere in die Niere. Arch. f. klin. Chirurgie Bd. 86 S. 399. 1908.

2) Coenen, Über Nebennierenverpflanzung. Arch. f. klin. Chirurgie Bd. 81 IJ. 2 S. 288. 
eine äusserst feine Naht das Kapselparenchym der Niere lose befestigt, um einen zu grossen Druck von seiten des Nierengewebes zu vermeiden. Manchmal wurde auch ein der Nebenniere entsprechendes Stückchen der Niere ausgeschnitten und die erstere an diese Stelle verpflanzt und fixiert. Die ganze Prozedur ist einfach und mit der linken Nebenniere in 10-15 Minuten zu beendigen.

Alle Tiere überlebten diesen operativen Eingriff und wurden zum Zwecke der Untersuchung nach verschieden langer Zeit getötet. Dann wurde die Milz resp. die Niere mit der implantierten Nebenniere herausgenommen, die Hälfte der implantierten Nebenniere für die histologische Untersuchung konserviert, die andere Hälfte zum Nachweis von Adrenalin nach der Methode von Meltzer-Ehrmann verwendet. $\mathrm{Zu}$ diesem $\mathrm{Zwecke}$ wurde das Organ mit Kochsalz extrahiert oder die Schnittfäche auf ein enukleiertes Froschauge angelegt. Wenn sich die Pupille dieses Auges nicht erweiterte, so wurde es mit einer gesunden Nebenniere oder Adrenalin-Takamine nachgeprüft und seine Reaktionsfähigkeit konstatiert; es fand́ sich kein richtig ausgeschnittenes Auge, das nicht auf Adrenalin oder eine normale Nebenniere reagiert hätte.

Im ganzen verfüge ich über 16 Versuche der oben geschilderten Art, von denen 14 an Kaninchen und 2 an Katzen ausgeführt wurden; die Prüfung der Nebenniere auf ihren Adrenalingehalt erfolgte bei je einem Tier 20 resp. 24 Stunden nach der Verpflanzung derselben; bei drei Tieren nach 48 Stunden, bei zwei Tieren nach 3 Tagen, bei einem Kaninchen nach 4 Tagen, bei zwei Tieren nach 1 Woche, bei je einem Tiere nach 10 Tagen, 4 und 7 Wochen und endlich bei 2 Tieren nach 10 Wochen und bei einem nach 17 Wochen.

Dabei zeigte sich, dass die in die Niere und Milz transplantierte Nebenniere nach 20-24 Stunden eine deutliche Adrenalinreaktion erkennen lässt.

In zwei Fällen, bei denen je ungefähr ein Viertel der Nebenniere in die Milz und Niere transplantiert war, ergab das in die Milz transplantierte Stück nach zweimal 24 Stunden keine Adienalinreaktion, während das in die Niere verpflanzte nach derselben Zeit noch eine deutliche, allerdings minimale und verspätete Reaktion zeigte. Bei direkter Applikation der gesunden Nebenniere auf ein Froschauge vergrössert sich die Pupille schon nach 5 Min. und ist nach 15 Min. maximal erweitert. Bei diesem Falle zeigte die Pupille erst nach 1 Stunde eine geringe Erweiterung. In einem Falle, bei 
dem die ganze Nebenniere in die Milz verpflanzt wurde, zeigte sich nach derselben Zeit noch eine minimale Reaktion. Nach dreimal 24 Stunden zeigte keine verpflanzte Nebenniere eine Adrenalinreaktion.

Das makroskopische Bild der in die Milz eingepflanzten Nebenniere zeigt folgendes Verhalten: Nach 24 Stunden erscheint die mit mehr oder weniger Blutcoagulis umgebene Nebenniere sehr wenig verändert und sieht auf der Schnittfläche etwas matt aus; die Konturen der Mark- und Rindenschicht sind deutlich erkennbar. Sie ist mit der Milz ziemlich gut verklebt, aber noch sehr leicht herauszulösen. Nach zweimal 24 Stunden ist die etwas voluminös gewordene Nebenniere auf der Schnittlläche weisslich-rosa, matt; gelegentlich ist die Grenze zwischen Rinde und Mark noch erkennbar.

Vier Tage bis 1 Woche nach der Transplantation ist die Nebenniere deutlich vergrössert und erscheint grauweiss (nekrotisch); in einem Falle war die Nebenniere noch 7 Tage nach der Transplantation anscheinend gut erhalten, so dass man hätte an ihre Lebensfähigkeit glauben können. Nach 4 Wochen ist das transplantierte Organ klein; aber gelegentlich sieht man noch nach 10-17 Wochen verkleinerte, aber in Form und Kontur der Rinde und des Marks anscheinend wohlerhaltene Nebennieren.

Das makroskopische Aussehen der in die Niere eingepflanzten Nebenniere ist im grossen und ganzen mit dem Bilde der in rie Milz transplantierten identisch, nur ist diese bei der ersteren häufiger deutlich nekrotisch als bei der letzteren.

Bei der histologischen Untersuchung der in die Milz inplantierten Nebenniere findet man schon nach 24 Stunden die Zellen etwas aufgequollen und schlecht färbbare Kerne. Die Marksubstanz lässt sich nicht mehr chromieren. Um die Nebenniere, im Mutterboden der Milz oder zwischen der eingepflanzten Nebenniere, sieht man ein geringeres oder grösseres Quantum Blut und Leukocyten; letztere dringen auch entlang der Nebennierengefässe verschieden tief in die Rinde hinein.

Nach zweimal 24 Stunden ist das Bild von dem vorher geschilderten nicht sehr wesentlich verschieden; die Zellkonturen in der Marksubstanz und den zentralen Rindenpartien sind oft verwischt.

Nach 3 Tagen bilden sich schon an einzelnen Stellen Erweichungszysten, während andere Teile, besonders die Rindenabschnitte, noch ziemlich gut erhalten scheinen. 
Die Präparate vom 4 . bis 7 . Tage zeigen schon oft vollständige Nekrose, indem sich die Kerne nicht mehr mit kernfärbendem Farbstoff färben lassen, während die Konturen der Kerne und die Parenchymstruktur oft sichtbar sind, welche noch längere Zeit erhalten bleiben können. Andere aus der gleichen Zeit haben noch ziemlich gut färbbare Kerne mit meist hellerem Zelleib.

Während eine Nebenniere 4 Wochen nach der Transplantation eine vollständige Nekrose erkennen liess, zeigte eine solche nach 10 Wochen das typische Bild von sogenannten "regressiven Zelleu" [Stoerk und v. Haberer $\left.{ }^{1}\right)$.

Bei diesem Präparat ist die Nebenniere durch eine dünne Bindegewebsschicht von der Milz getrennt. Das Protoplasma dieser regressiven Zellen ist leicht gelb gefärbt und hat ungleichmässig grosse nicht färbbare Tröpfchen in reichlicher Menge, so dass die Zellen ein helleres Aussehen bekommen. Die mehr zackigen Kerne lassen sich noch gut färben. Man sieht sehr viele grosse Zellen mit mehreren Kernen; die meisten davon scheinen durch Konfluenz von mehreren Zellen entstanden zu sein, weil man innerhalb der verschieden grossen mehrkernigen Zellen Andeutungen von Zellkonturen bemerkt. Das ganze Präparat ist von spiessigen Kristallen oder, genauer gesagt, von Lücken durchsetzt, die die Konturen der Kristalle erkennen lassen, welche durch die Behandlung selbst extrahiert worden waren. Sie sind schon in den Nebennieren 24 Stunden nach der Implantation zu sehen, wenn auch ihre Zahl nicht sehr gross ist. Man sieht ausserdem noch eine Ablagerung von Kalk, was auch in einem früheren Stadium zu beobachten ist. Bei einem Tier, das 17 Wochen nach der Operation getötet wurde, ist die implantierte Nebenniere von ziemlich starken Bindegewebszügen durchzogen, und zwischen den Maschen derselben sind teils nekrotische Zellverbände, teils Gruppen von regressiven Zellen mit Kristallen und Kalk zu sehen.

Im ganzen und grossen unterscheidet sich die in die Niere implantierte Nebenniere histologisch nicht wesentlich von der in die Milz implantierten; die in die Niere eingepflanzte Nebenniere lässt in den Anfangsstadien keine so starke Einwanderung der Leukocyten erkennen als wie die in die Milz implantierte Drüse.

1) Stoerk und v. Haberer, Verhandlungen der deutschen Gesellschaft f. Chirurgie 1908. 
Was nun die Funktion der transplantierten Nebenniere anbelangt, so baben die Versuche ergeben, dass die verpflanzte Drüse sehr bald ihre Tätigkeit einstellt, und dass in allen Fällen bereits nach 3 Tagen das Produkt dieser Tätigkeit, das Adrenalin, nicht mehr nachzuweisen ist. Es ist sehr wahrscheinlich, dass die Nebenniere unmittelbar nachdem sie aus ihrem normalen Zusammenhang mit dem Organismus gebracht wird, eine Einbusse ihrer physiologischen Funktion erleidet, und dass sie an dem Ort ihrer Einpflanzung überhaupt ihre normale Funktion nicht mehr aufnimmt. Die Tatsache, dass in der verpflanzten Drüse sich noch nach $2 \times 24$ Stunden Adrenalin nachweisen lässt, ist kein Beweis dafür, dass die Drüse auch Adrenalin am Ort ihrer Einpflanzung produziert hat, sondern es ist eher anzunehmen, dass dieses Adrenalin noch von der Tätigkeit der Drüse herrührt, bevor sie verpflanzt wordèn ist. Dass die Nebenniere ihre Tätigkeit am Ort der Einpflanzung sehr bald einstellt, geht unter anderem auch daraus hervor, dass die Marksubstanz, von der allgemein angenommen wird, dass sie Adrenalin liefert, schon nach 24 Stunden nicht mehr chromierbar ist. Es kann also das Adrenalin, das man noch am 2. Tage nach der Transplantation in der Nebenniere findet, nicht von dieser Marksubstanz sezerniert worden sein.

Es wäre nun denkbar, dass die Rindensubstanz dieses Adrenalin gebildet hat.

Nun sind fast alle Autoren darüber einig, dass die Rinde wohl kein Adrenalin bildet, und auch die eigenen Befunde erlauben nicht, den Schluss zu ziehen, dass die in der transplantierten Nebenniere erhalten gebliebene Rinde das Adrenalin geliefert haben könnte. Wenn auch die Rindensubstanz, die, wie es scheint, eine grössere Resistenz ausfweist als die Marksubstanz, in den ersten Tagen nach der Verpfianzung sich histologisch nicht sehr von der Rinde einer normalen Nebenniere unterscheidet, so dürfte auch sie sehr bald ihre spezifische Tätigkeit einstellen, wenn auch hierfür keine solchen Beweise vorliegen wie bei der Marksubstanz.

Dass aber die Rinde das Adrenalin nicht produziert, geht daraus mit Sicherheit hervor, dass ihr Aussehen am 3. Tage nach der Verpflanzung, wo kein Adrenalin mehr nachweisbar ist, sich nicht wesentlich unterscheidet von jenem vom 2. Tage. Und selbst Tage und Wochen, nachdem die Nebenniere verpflanzt wurde, ist das histologische Bild der Rinde ähnlich jenem vom 1. bis 3. Tage nach 
Über das Schicksal und die Funktion der transplantierten Nebenniere. 439

der Verpflanzung, und doch findet man niemals Adrenalin in solchen Nebennieren, was man wohl finden müsste, wenn die Rinde die Fähigkeit hätte, es zu bilden.

Man wird also annehmen müssen, dass das 48 Stunden nach der Verpflanzung in der Drüse vorhandene Adrenalin von der Marksubstanz geliefert worden ist, und zwar zum grössten Teil, ehe sie noch verpflanzt wurde, und zum geringen vielleicht unmittelbar nachdem sie in ein anderes Gewebe übertragen wurde, und dass es in dem Organ aufgespeichert bleibt, bis es in den Saftstrom des Organismus aufgenommen wird.

Dass dem wirklich so ist, dafür spricht die Beobachtung, dass das Adrenalin in einer Nebenniere, die in die Niere verpflanzt wurde, noch nach 48 Stunden nachweisbar war, während nach der gleichen Zeit eine in die Milz verpflanzte Nebenniere das Adrenalin nicht mehr erkennen lässt.

Dafür spricht ferner die Beobachtung, dass kleine, in die Milz übertragene Stückchen früher das Adrenalin verlieren als die ganze Nebenniere. Gleichgrosse Stückchen behalten in der Niere länger das Adrenalin als in der Milz, offenbar weil das eingeführte Nebennierenstückchen in dem strafferen Nebennierengewebe infolge des schlechteren Kontaktes erst später in den Saftaustausch tritt als in dem allseits gut zugänglichen Milzgewebe. Diese Vermutung wird ferner gestützt durch die Tatsache, dass zu Beginn der Transplantation die Einwanderung der Leukocyten in der in die Niere implantierten Nebenniere viel geringer ist als in der in die Milz übertragenen. Es ist wahrscheinlich, dass die zarten Nebennierenzellen nach der Trennung von der Gefässverbindung nicht lange ihre Funktion bewahren können, und dass das früher produzierte und im Innern erhaltene Adrenalin allmählich von den umgebenden kommunizierenden Saftkanälen resorbiert wird ${ }^{1}$ ).

Um in ähnlicher Weise das Schicksal der aus ihren Verbindungen losgetrennten, aber nicht in andere Organe transplantierten, sondern in loco gelassenen Nebenniere zu studieren, habe ich in sechs Fällen die linke Nebenniere vorsichtig lospräpariert und in loco gelassen. Bei einem von diesen sechs Tieren wurde die Nebenniere 24 Stunden

1) Wenn man in einem getöteten Tiere eine Nebenniere von der Umgebung trennt und in loco lässt, behält sie nicht weniger als 8 Tage das Adrenalin, auch wenn Fäulnis eintritt. 
nach der Operation, bei zwei nach zweimal 24 Stunden und bei je einem Tiere nach 3 und 7 Tagen herausgenommen und wie bei den vorigen Versuchen der Adrenalingehalt derselben an einem frisch herausgeschnittenen Froschauge untersucht. Die von dem umgebenden Gewebe und Organen, besonders von Mesenterium- und Dünndarmschlingen, anfangs ganz lose umbüllte und nach einer Woche schon ziemlich fest damit verwachsene Nebenniere zeigt nur in den ersten 24 Stunden die Adrenalinreaktion; nach zweimal 24 Stunden und später schon keine mehr. Die mikroskopische Untersuchung liess immer deutliche Zeichen eines nekrotischen Unterganges des Nebennierengewebes erkennen.

Es ist begreiflich, dass in diesen Fällen die anfangs in der lockeren retroperitonealen Bindegewebswunde und dem grossen Lymphraum der Peritonealhöhle liegende und später mit dem umgebenden Gewebe verwachsende Nebenniere sehr leicht ihres Inhaltes verlustig geht und das Adrenalin früher verliert als in dem Milzund Nierengewebe.

In einer weiteren Versuchsreihe wurde die Nebenniere bis auf den Gefässstiel aus ihrer Umgebung herausgeschält, in der Absicht, die nervösen Verbindungen nach Möglichkeit zu zerstören. Der Zweck dieser Versuche war wesentlich der, zu sehen, ob bei Ausschaltung des Nerveneinflusses die Nebenniere ihre Adrenalinproduktion fortsetzt.

Da die feinen Nerven, die mit dem Bindegewebe um die Gefässe in das Organ hineintreten, erhalten bleiben, war der eigentliche Zweck nicht vollständig zu erreichen, doch möchte ich immerhin den Ausgang dieser Versuche kurz mitteilen.

Es wurde zuerst bei fünf Kaninchen die linke Nebenniere von dem umgebenden Gewebe losgetrennt und nur mit dem medianen fadendünnen Gefässtiel in loco gelassen; die rechte Nebenniere wurde dabei nicht berührt. Je ein Kaninchen wurde 24 Stunden, zweimal 24 Stunden, 3, 7 und 9 Tage nach dem Eingriff getötet und die linke Nebenniere zur Untersuchung herausgenommen. Sie zeigte je nach der Grösse und Lokalisation des Stieles verschieden grosse und geformte Reste von gesundem Gewebe, die sich schon nach 3 Tagen ziemlich gut von den nekrotischen demarkierten; alle zeigten deutliche Adrenalinreaktion.

Um $z \mathfrak{u}$ entscheiden, ob das Adrenalin in der nur mit Gefässstiel belassenen Nebenniere aus der anderen Nebenniere stammt, wurde 
in weiteren Versuchen die rechte Nebenniere vollständig von der Umgebung losgetrennt, während die linke mit dem Gefässstiel belassen wurde. Alle Tiere überlebten diesen Eingriff, und wurden zwei davon 24 Stunden nach der Operation, je eines zwei- und dreimal 24 Stunden, zwei nach 5 und eines nach 10 Tagen getötet. Die rechte Nebenniere zeigte die Adrenalinreaktion nur in den ersten 24 Stunden. Die mit dem Gefässstiel belassene linke Nebenniere zeigte stets die Adrenalinreaktion, und liessen sich dementsprechend makroskopisch und histologisch dem zurückgelassenen Gefäss entsprechende kleine Reste gesunden Nebennierengewebes nachweisen. Dabei zeigte sich, dass Tiere, bei denen einseitig ganz kleine Stückchen von Nebenniere, die bald hauptsächlich aus Marksubstanz (mit schöner ringförmiger Rindennekrose mit Ausnahme des Stielteiles), bald anscheinend nur aus Rindensubstanz bestehen, ohne besondere Erscheinungen wenigstens durch 6-10 Tage am Leben bleiben.

\section{Anhang.}

Da alle meine Versuche in Äthernarkose ausgeführt wurden und eine solche nach Schur und Wiesel ${ }^{1}$ ) eine Ausschwemmung von Adrenalin aus der Nebenniere bewirken soll, so habe ich diese Angabe der genannten Autoren einer Nachprüfung unterzogen, über die ich hier anhangsweise berichten möchte, obgleich dies zu meinem eigentlichen Thema in keiner Beziehung steht.

Ich habe sechs Kaninchen verschieden lange (3-6 Stunden) mit Äther teils durch subkutane Injektion, teils durch Inhalation tief narkotisiert, so dass bei drei derselben wegen Scheintod öfters künstliche Atmung ansgeführt werden musste. Während der Narkose wurde Blut vom Ohr oder von der Femoralis, nach dem Torle vom Herzen entnommen und am Froschauge untersucht; beim Vergleich mit gesunden Tieren ergab sich kein Unterschied. Der Nebennierenextrakt übte nach verschieden langer Narkose, bis zu 6 Stunden, eine ganz prompte Wirkung aus.

In meinen Versuchen konnte ich demnach einen Einfluss der Narkose auf die Adrenalinproduktion nicht nachweisen, und es lässt sich keineswegs behaupten, dass ein Verschwinden der Adrenalin-

1) Schur und Wiesel, Über das Verhalten des chromaffinen Gewebes bei der Narkose. Wiener klin. Wochenschr. 1908 Nr. 8. 
reaktion in der Nebenniere nach fünf- oder sechsstündiger tiefer Narkose bei Kaninchen immer nachweisbar ist.

Die oben beschriebenen Tatsachen lassen sich in folgenden Sätzen resümieren.

1. Eine in die Milz oder Niere transplantierte Nebenniere lässt nach zweimal 24 Stunden Adrenalin erkennen.

2. Nach dieser Zeit verschwindet das Adrenalin, und zwar bei einer in die Milz transplantierten Nebenniere früher als bei einer in die Niere transplantierten.

3. Während die Rinde ziemlich lange Zeit (über 10-17 Wochen) in leidlichem Zustande erhalten bleiben kann, verschwindet die Chromierbarkeit der Marksubstanz schon nach 24 Stunden.

4. Eine in loco belassene, aber aus ihrer Verbindung mit ihrer Umgebung getrennte Nebenniere verliert das Adrenalin schon nach 24 Stunden.

5. Die transplantierte Nebenniere stellt wahrscheinlich unmittelbar nach ibrer Verpflanzung ihre Tätigkeit ein.

6. Das in der transplantierten Nebenniere 48 Stunden nach der Verpflanzung nachweisbare Adrenalin ist das Produkt der Tätigkeit dieser Drüse vor der Transplantation.

7. Das mehr oder weniger rasche Verschwinden dieses Adrenalins hängt von der Beschaffenheit des Gewebes $a b$, in welches die Nebenniere transplantiert wurde.

8. Die Adrenalinsekretion scheint von nervösen Einflüssen in weitem Maasse unabbängig zu sein.

9. Der von Schur und Wi esel angegebene Einfluss der Narkose auf die Adrenalinsekretion besteht keineswegs für alle Tiere zu Recht.

Zum Schlusse erfülle ich die angenehme Pflicht, Herrn Prof. Dr. Alois Kreidl für die Anregung und Unterstutzung bei der Durchführung dieser Arbeit auch an dieser Stelle bestens zu danken. 